\title{
Emprego de Óleos Essenciais como Matéria-Prima para a Produção de Repelentes de Insetos
}

\author{
Angélica Gomes Coelho ${ }^{1}$, Ezequielina Raquel Leal ${ }^{2}$, \\ Jeany Francisca Sampaio de Vasconcelos ${ }^{3}$
}

\begin{abstract}
RESUMO
Os óleos essenciais extraídos de plantas são comuns e popularmente empregados como alternativa aos repelentes sintéticos comercializados. Assim, o presente trabalho propõe-se a realizar uma revisão na literatura sobre o emprego de óleos essenciais na análise e desenvolvimento de formulações repelentes. Realizou-se uma busca por artigos científicos usando os descritores "Repellent*", "Plant*", "Essential* oil*" e "Cosmetic*", nas bases de dados SciELO e PubMed". As publicações selecionas foram analisadas quanto ao país de origem, famílias mais estudadas, partes das plantas mais usadas, métodos de extração empregados e tipo de formulações repelentes desenvolvidas. A busca resultou em um total de 241 artigos científicos, em sua maioria do ano de 2015. Encontraram-se espécies das mais diferentes famílias, destacando-se a Asteraceae. Além disso, a maioria das publicações é originária da China e do Brasil, sendo a folha a parte da planta mais utilizada e a hidrodestilação o método extrator mais prevalente. A representativa pesquisa acerca dos óleos vegetais repelentes, contudo, está associada à divulgação de apenas cinco formulações cosméticas repelentes, as quais possivelmente teriam maior segurança de uso e menor valor no mercado. Assim, mediante os estudos científicos analisados, conclui-se que o mercado cosmético brasileiro carece do aproveitamento completo das propriedades investigadas e comprovadas para óleos essenciais repelentes de insetos, sendo este um campo promissor para o desenvolvimento de novos produtos.
\end{abstract}

Palavras-chave: Repelentes. Cosméticos. Óleos essenciais. Planta.

\section{EMPLOYMENT OF ESSENTIAL OILS AS RAW MATERIAL FOR THE PRODUCTION OF INSECT REPELLENTS}

\section{ABSTRACT}

Essential oils extracted from plants are common and popularly used as an alternative to marketed synthetic repellents. Thus, the present work proposes to carry out a systematic review in the literature on the use of essential oils in the analysis and development of repellent formulations. A search for scientific articles using the descriptors "Repellent *", "Plant *", "Essential * oil *" and "Cosmetic *" was carried out in the databases: SciELO and PubMed ${ }^{\circledR}$. Selected publications were analyzed for country of origin; families studied; parts of plants most employed; extraction methods employed and type of repellent formulations developed. The search resulted in a total of 241 articles, mostly from the year 2015. We found species of the most different families, especially the Asteraceae. In addition, most of the publications originate in China and Brazil, with the leaf being the most used plant part and hydrodistillation the most prevalent extraction method. However, representative research on repellent vegetable oils is associated with the disclosure of only five cosmetic repellent formulations, which would possibly have greater safety of use and lower market value. Thus, through the scientific studies analyzed, it is concluded that the Brazilian cosmetic market needs to take full advantage of the investigated and proven properties for insect repellent essential oils, which is a promising field for the development of new products.

Keywords: Insect repellents. Cosmetics. Oils. Plant.

RECEBIDO EM: 7/3/2019

MODIFICAÇÕES REQUERIDAS EM: 5/7/2019

ACEITO EM: 16/8/2019

\footnotetext{
Graduada em Farmácia. Mestre em Ciências Farmacêuticas pela Universidade Federal do Piauí. Especialista em Gestão da Assistência Farmacêutica pela Universidade Federal de Santa Catarina. Como pesquisadora, tem experiência nas áreas de Química de Produtos Naturais e Tecnologia de Medicamentos. Possui experiência profissional em farmácia comunitária, magistral e hospitalar, além da atuação como docente do curso de Farmácia da Faculdade Integral Diferencial (Facid Wyden). angelicacoelho13@gmail.com

2 Graduanda em Farmácia. Monitora Teórica de Farmacologia. Membro da Liga Acadêmica de Farmacologia do Piauí. ezeeraquel16@gmail.com

3 Graduação em Farmácia pela Faculdade Integral Diferencial, Brasil (2018). Técnica de enfermagem do hospital municipal e maternidade de Coelho Neto, Brasil. jeannysamppaiovas@hotmail.com
} 


\section{INTRODUÇÃO}

As doenças causadas por patógenos veiculados por artrópodes sugadores de sangue têm uma importância fundamental sobre a saúde pública. Segundo a Organização Mundial da Saúde (WHO, 2013), 3,3 biIhões de pessoas ainda vivem onde a malária é uma ameaça constante, e 3.900 milhões de pessoas correm risco de contrair o vírus da dengue juntamente com novos surtos de arbovírus, entre os quais chikungunya e zika vírus, que, recentemente, recebeu grande ênfase (BENELA; PAVELI, 2018).

Diante disso, os repelentes surgem como substâncias químicas naturais ou sintéticas que, quando aplicadas no vestuário ou na pele, impedem a aterrissagem do mosquito e, consequentemente, a picada, reduzindo, assim, o risco de transmissão de doenças veiculadas por esses insetos, assim como as reações imunoalérgicas resultantes (TAVARES et al., 2018).

A RDC 19, de 10 de abril de 2013, estabelece os requisitos técnicos mínimos relativos à segurança, à eficácia e à rotulagem para a concessão de registro de produtos cosméticos repelentes de insetos. A norma destaca as formulações à base de DEET ( $N$, N-DIETIL-META-TOLUAMIDA E N,N-DIETIL-3-METILBENZAMIDA), um ativo sintético de amplo espectro, eficaz e persistente na pele, cujo emprego no produto repelente implica cuidados específicos quanto à concentração utilizada e quanto ao público-alvo (BRASIL, 2013). Neste sentido, ressalta-se ainda a necessidade do uso de produtos repelentes durante a gestação, em razão dos danos neurológicos ao feto causados pela infecção pré-natal por zika vírus, sendo imprescindível, portanto, o uso de ativos repelentes seguros e eficazes para este público-alvo (WYLIE et al., 2016).

Os óleos essenciais extraídos de plantas são um dos produtos de baixo custo mais comuns e amplamente utilizados como alternativa aos repelentes sintéticos comercializados. São considerados mais seguros devido a uma longa história de utilização para consumo humano nas fragrâncias e medicamentos. Diferentes famílias de plantas demonstraram ter extratos com atividades de mosquitocida contra diferentes espécies de vetores (CHELLAPPANDIAN et al., 2018).

Considera-se, portanto, a necessidade de incentivar a inserção de novos repelentes no mercado cosmético, uma vez que esta classe de produtos é de uso imprescindível como medida de prevenção às infecções de grande repercussão em saúde pública, transmitidas por mosquitos hematófagos, tais como dengue, zika vírus e chikungunya. Nesse contexto, o presente trabalho tem como objetivo realizar um estudo de revisão na literatura sobre o emprego de óleos essenciais no desenvolvimento de formulações repelentes de insetos.

\section{METODOLOGIA}

Realizou-se uma busca por artigos científicos usando os descritores "Repellent*", "Plant", "Essential* oil*" e "Cosmetic*", nas bases de dados Biblioteca Científica Eletrônica em Linha (Scientific Electronic Library On-line ou SciELO) e PubMed ${ }^{\circledR}$ via National Library of Medicine, com consulta do Medical Subject Headings (MeSH), sendo selecionadas para análise as publicações entre os anos de 2013 e 2017, excluindo-se do objeto de estudo os artigos de revisão ou os que não tratavam do uso de óleos essenciais nas formulações repelentes como assunto principal.

Na pesquisa foram analisadas as datas e os países com maiores quantidades de artigos publicados, famílias de espécies vegetais mais usadas nas formulações repelentes, partes das plantas mais utilizadas para o determinado fim, principais métodos de extração empregados para obtenção dos óleos essenciais e tipo de formulações repelentes desenvolvidas. Por fim, os resultados foram expressos na forma de tabelas e gráficos, construídos mediante o software Microsoft Office Excel 2010.

\section{RESULTADOS E DISCUSSÃO}

A busca por repelentes à base de substâncias naturais surgiu a partir da observação de que os compostos sintéticos, especialmente à base do composto DEET, apresentavam odor desagradável, sensação gordurosa na pele e poderiam provocar reações tóxicas em crianças, resultando em baixa aceitação do usuário. Além disso, sabe-se que, antes do uso de substâncias sintéticas, os humanos já utilizavam plantas para se proteger contra insetos hematófagos (NORASHIQIN; ZURAINEE; ROHANI, 2016).

Desse modo, a busca por pesquisas, vislumbrando a aplicabilidade de óleos vegetais no desenvolvimento de novos repelentes, sumariza uma demanda do mercado cosmético, quando há avidez por produtos atóxicos e de baixo custo. Assim, esta revisão de literatura resultou em um total de 241 artigos científicos, sendo 187 referentes à base PubMed e 54 ao Scielo. Foi observado nesta análise que no ano de 2015 houve o maior número de publicações, seguido de 2017. 
Os óleos essenciais (OEs) são definidos como substâncias complexas voláteis, lipofílicas, geralmente odoríferas e líquidas, oriundas do metabolismo secundário de vegetais. Estes podem ser aplicados em vários segmentos, como antibacterianos, antivirais, antifúngicos, inseticidas e contra o ataque de herbívoros, bem como nos setores de higiene pessoal, perfumaria e cosmética (MILLEZI et al., 2014).

Esses óleos não são moléculas simples, e sim uma ampla variedade de compostos, com diversas funções e mecanismos de ação. Inúmeros princípios ativos de determinado óleo essencial podem ser encontrados em diferentes plantas, e, consequentemente, em diversas concentrações, como o óleo essencial de tomilho, que possui em sua composição $40 \%$ de timol, em contrapartida o óleo essencial de orégano, que também possui esse constituinte, porém em 10\% de sua composição total. Dessa maneira, dependendo do composto em maior concentração, determina-se o modo de ação e função de cada óleo essencial. Além disso, é sabido que o sinergismo entre óleos essenciais ou entre seus princípios ativos pode potencializar seus efeitos (RODRIGUES et al., 2017).

Considerando-se a aplicabilidade destes constituintes como repelentes de insetos, destaca-se o uso do óleo essencial de Citronela, cujo mecanismo de ação baseia-se na promoção de uma intensa excitação do sistema nervoso central do inseto e um bloqueio da circulação de sódio nas células nervosas por meio da inibição do trifosfato de adenosina, acetilcolinesterase e do receptor ácido $\gamma$-amino butírico (Gaba), provocando uma paralisia no inseto (CARNEIRO, 2015).

Em relação aos países de origem dos artigos selecionados para esta revisão, como pode ser observado na Figura 1, percebe-se que grande parte das publicações é asiática, destacando-se a China com 28 artigos, a Índia com 15 e a Tailândia com 9. Merece destaque também a produção brasileira sobre este tema, totalizando 22 artigos, diretamente relacionados ao possível desenvolvimento de um produto repelente à base de óleos vegetais.

A China destaca-se pela sua medicina tradicional, proveniente das mais antigas civilizações, ainda vivas, que utilizam fitoterápicos e outras substâncias de origem natural. Por outro lado, o Brasil possui posição privilegiada em biodiversidade e utiliza-se deste fato para investigar e extrair as propriedades terapêuticas de diversas espécies naturais, uma vez que em território brasileiro coexiste a maior quantidade total de espécies catalogadas no mundo (13\%), além de tra- tar-se da nação com a segunda maior quantidade de espécies endêmicas do mundo, em valores absolutos (PIMENTEL et al., 2015).

Figura 1 - Quantidade de artigos detectados por país de origem dos estudos

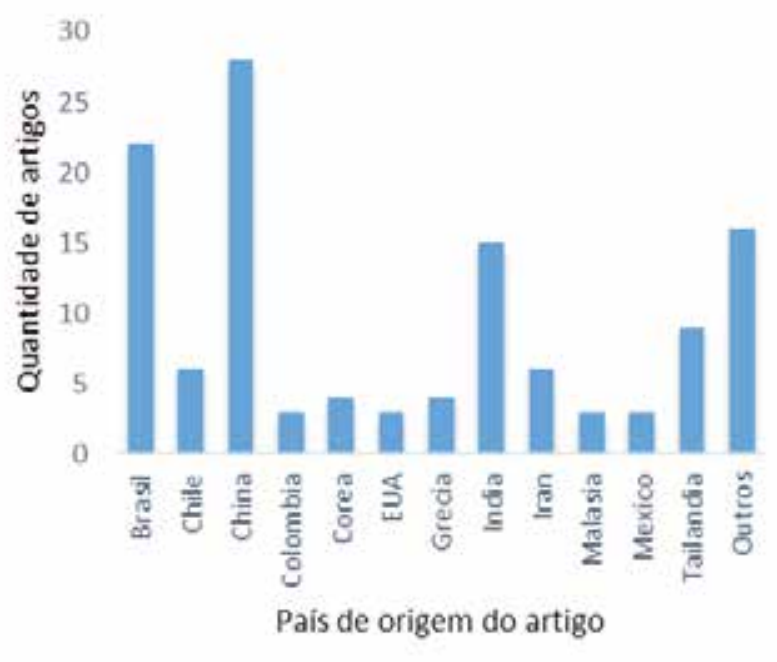

Fonte: Dados da pesquisa.

Na busca por espécies investigadas como repelentes naturais, foram encontradas espécies das mais diferentes famílias, conforme mostra a Figura 2, destacando-se a Asteraceae, cujos representantes apareceram em 24 estudos, seguida da Lamiaceae e Rutaceae, com suas espécies presentes em 18 estudos.

Figura 2 - Distribuição das principais famílias das espécies vegetais empregadas nas pesquisas científicas sobre óleos essenciais com atividade repelente

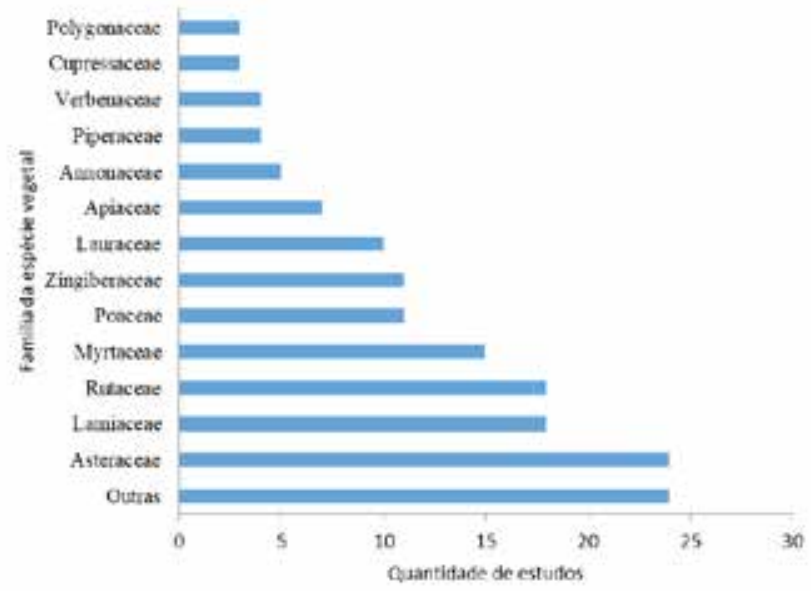

Fonte: Dados da pesquisa.

Nos estudos encontrados observou-se que os óleos essenciais de espécies vegetais da família Asteraceae demonstraram efeito repelente contra os insetos Culex quinquefasciatus, Aedes aegypti e Triatoma 
infestans, vetores das doenças filariose, dengue/zika/ chikungunya e doença de chagas, respectivamente (GUERREIRO et al., 2018).

Além disso, estudos demonstraram que os óleos essenciais que possuem os melhores resultados como repelentes são: citronela (Cymbopogon nardus), cravo (Syzygium aromaticum), verbena (Verbena officinalis), cedro (Cedrella fissilis), lavanda (Lavandula angustifólia), pinho (Pinus sylvestris), canela (Cinnamomum verum), alecrim (Rosmarinus officinalis), manjericão (Ocimum basilicum), pimenta e pimenta da Jamaica (FÁVERO, 2014).

De acordo com a Figura 3, verificou-se que a foIha foi a parte da planta mais utilizada para a extração do óleo essencial com propriedade repelente. Sabe-se que o tipo de material vegetal (folhas, flores, caules ou raízes), bem como o estágio de desenvolvimento do órgão, são dois fatores determinantes da composição do óleo essencial. As flores, folhas e caules são os órgãos vegetais onde, com maior incidência, encontram-se estruturas secretoras particulares (tricomas, canais ou bolsas) por meio das quais são produzidas misturas complexas de produtos naturais. Dependendo da espécie, existem ainda outros órgãos vegetais, igualmente aromáticos, que podem ser, consoante a finalidade, consumidos em verde, ou depois de secos, ou dos quais se podem extrair óleos essenciais utilizados em aplicações diversas (FIGUEIREDO; PEDRO; BARROSO, 2014).

Figura 3 - Distribuição das principais partes vegetais empregadas nas pesquisas científicas para obtenção de óleos essenciais com efeito repelente

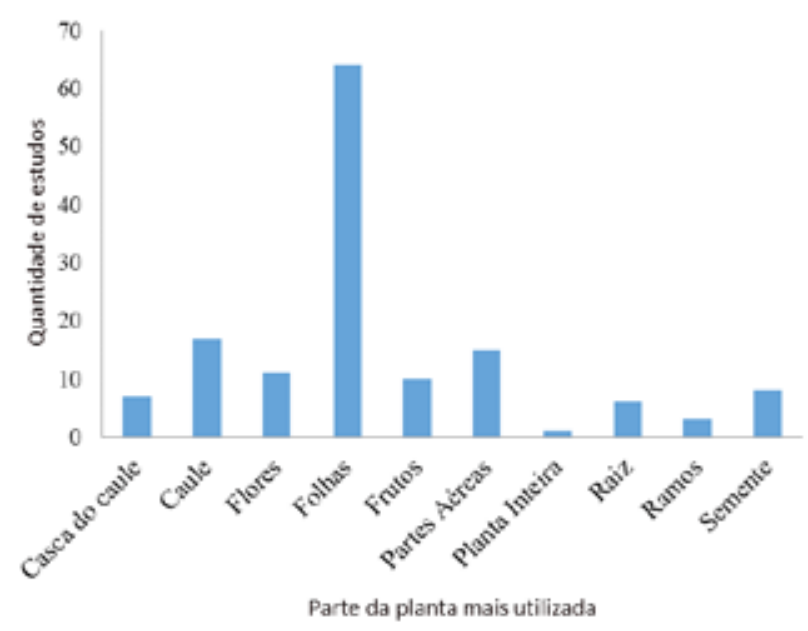

Fonte: Dados da pesquisa
Em relação ao método extrator, o mais utilizado foi a hidrodestilação com $91,6 \%$, seguida de destilação a vapor com $7,29 \%$ e soxhlet com $1,11 \%$, conforme mostra a Figura 4.

Figura 4 - Distribuição dos principais métodos de extração empregados nas pesquisas científicas para obtenção de óleos essenciais

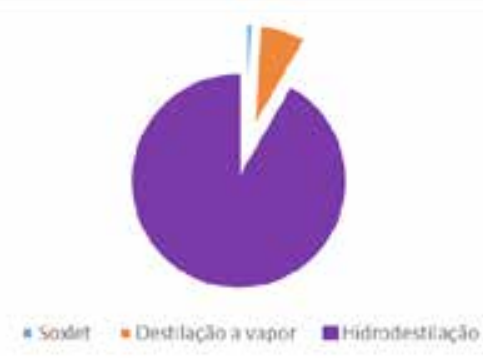

Fonte: Dados da pesquisa.

Os métodos de extração dos óleos voláteis variam conforme a sua localização na planta e também da utilização do mesmo (DANH et al., 2013). O método de hidrodestilação consiste na separação dos componentes voláteis em virtude da pressão de vapor mais elevada que a da água, portanto são arrastados pelo vapor d'água. Este é um método muito simples, fácil de operar, de baixo custo e a proporção extraída de óleo essencial pode chegar a rendimento bastante elevado (LEÃO, 2015).

Esta revisão de literatura revelou a ocorrência de apenas cinco artigos que tratam diretamente sobre o desenvolvimento de formulações cosméticas repelentes de insetos, utilizando óleos essenciais, sendo os dados das formulações sumarizados no Quadro 1.

No desenvolvimento de produtos repelentes, sabe-se que a eficácia dos ingredientes ativos é influenciada pela formulação, a qual regula as taxas de evaporação e tempos de proteção dos ativos, afetando a persistência do repelente. A utilização de emplastros permite a liberação contínua e controlada dos óleos, assim como nas microencapsulações, que protegem os óleos essenciais de volatilizarem rapidamente, pois estão presos em uma estrutura complexa. A otimização da formulação repelente, portanto, é de suma importância para prolongar o efeito repelente de um óleo essencial (NERIO; OLIVERO-VERBEL; STASHENKO, 2010).

Desse modo, cabe destacar que os repelentes naturais, uma vez presentes no mercado farmacêutico, são obtidos de recursos renováveis e do desenvolvimento da resistência dos insetos a essas substâncias, compostas da associação de vários princípios ativos, o que é um processo lento. Esses compostos, todavia, são de fácil acesso e sua obtenção promove incentivos à cadeia agrícola nacional, representando opção segura e de baixo custo de produção (FREIRE, 2017). 
Quadro 1 - Descrição dos produtos cosméticos repelentes desenvolvidos e divulgados por publicações de 2013 a 2017

\begin{tabular}{|c|c|c|c|c|}
\hline Óleo essencial (OE) & Parte utilizada & $\begin{array}{c}\text { Técnica de extração/ } \\
\text { obtenção }\end{array}$ & Produto desenvolvido & Referência \\
\hline OE de Piper aduncum L & Folhas & Hidrodestilação & Pomada, creme e gel & $\begin{array}{c}\text { MAMOOD et al., } \\
2016\end{array}$ \\
\hline $\begin{array}{c}\text { OE de Citrus aurantifolia, Citrus } \\
\text { grandis e Alpinia galanga. }\end{array}$ & $\begin{array}{c}\text { Folhas, casca de } \\
\text { frutos e rizoma. }\end{array}$ & Hidrodestilação & $\begin{array}{c}\text { OE microencapsulado } \\
\text { e incorporado a uma } \\
\text { base de loção }\end{array}$ & $\begin{array}{c}\text { MISNI; NOR; } \\
\text { AHMAD, 2014 }\end{array}$ \\
\hline $\begin{array}{c}\text { OE de Cinnamomum zeylanicum, } \\
\text { Cymbopogon citrullus }\end{array}$ & Cascas, folhas & Não informada & Emplastro & $\begin{array}{c}\text { CHATTOPADHYY } \\
\text { et al., 2015 }\end{array}$ \\
\hline OE de Citronella spp. & Não informada & Obtido & Creme & YADAV et al., 2014 \\
\hline $\begin{array}{c}\text { Mistura complexa de óleos } \\
\text { vegetais }\end{array}$ & Não informada & Obtido & $\begin{array}{l}\text { OE microencapsulado } \\
\text { e incorporado à base } \\
\text { come pomada }\end{array}$ & WU et al., 2013 \\
\hline
\end{tabular}

Fonte: Dados da pesquisa.

\section{CONCLUSÃO}

Há uma quantidade expressiva de artigos científicos que tratam da atividade de repelência a insetos desempenhada pelos óleos essenciais de origem vegetal, sendo estes, em sua maioria, da China e do Brasil. As principais espécies pertencem à família Asteraceae, sendo a folha a parte da planta mais utilizada e a hidrodestilação o método extrator mais prevalente. A representativa pesquisa acerca dos óleos vegetais repelentes, contudo, está associada à divulgação de apenas cinco formulações cosméticas, o que pode significar, para o mercado cosmético brasileiro, o aproveitamento incompleto ou tardio das propriedades investigadas e comprovadas por intermédio dos estudos científicos.

\section{REFERÊNCIAS}

BENELA, G.; PAVELI, R. Beyond Mosquitoes - Essential Oil Toxicity and Repellency Against Bloodsucking Insect. Revista Industrial Crops \& Products, v. 117, p. 382-392, Feb. 2018.

BRASIL. Ministério da Saúde. Agência Nacional de Vigilância Sanitária. Resolução da Diretoria Colegiada-RDC no 19, de 10 de abril de 2013.

CARNEIRO, W. V. Óleo essencial de citronela: avaliação do seu potencial como repelente veiculado em uma loção cremosa. 2015. Trabalho (Conclusão Graduação em Farmácia) - Universidade Federal da Paraiba, João Pessoa,. 2015.

CHELLAPPANDIAN, M. et al. Botanical essential oils and uses as mosquitocides and repellents against dengue. Environment Internacional, v. 113, p. 214-230, 2018.

DAHN, L. T et al. Antioxidant activity, yield and chemical composition of lavender essencial oil extracted by supercritical $\mathrm{CO}_{2}$. The Journal of Supercritical Fluids. v. 70, p. 27-34, 2013. FÁVERO, R. Estudo de repelência com diversos produtos de origem natural em operárias de Apis melifera em semi-campo. 2014. Trabalho (Conclusão de Bacharelado em Ciências Biológicas) - Universidade Estadual Paulista, Rio Claro,. 2014.
FIGUEIREDO, A. C.; PEDRO, L. G.; BARROSO, J. G. Plantas aromáticas e medicinais. Revista da Associação Portuguesa de Horticultura-APH, n. 124, p. 29-33, 2014.

FREIRE, N. F. Desenvolvimento de nanopartículas poliméricas contendo óleo essencial de citronela (Cymbopogon winterianus). 2017. Dissertação (Mestrado em Engenharia Industrial) - Universidade Federal da Bahia, Salvador, 2017. GUERREIRO, A. C. et al. Assessment of the Insecticidal Potential of the Eupatorium buniifolium Essential Oil Against Triatoma infestans (Hemiptera: Reduviidae). A Chiral Recognition Approac. Neotropical Entomology. v. 47, p. 418, 2018.

LEÃO, M. Análise do óleo essencial da laranja doce Citrus Sinensis (L.) Osbeck obtido das cascas secas e frescas através do método de extração por hidrodestilação. 2015. Trabalho (Conclusão de Graduação em Farmácia) - Universidade de Santa Cruz do Sul, Santa Cruz do Sul,. 2015.

MILLEZI, A. F et al. Caracterização química e atividade antibacteriana de óleos essenciais de plantas condimentares e medicinais contra Staphylococcus aureus e Escherichia coli. Revista Brasileira de Plantas Medicinais, Botucatu, v. 16, n. 1, 2014.

NERIO, L. S.; OLIVERO-VERBEL, J.; STASHENKO, E. Repellent activity of essencial oils: A review. Bioresource Technology. v. 101, p. 372-378, 2010.

NORASHIQIN, M.; ZURAINEE, M. N.; ROHANI, A. New Candidates for Plant- Based Repellents Against Aedes aegypti. Journal of the American Mosquito Control Association, v. 32, n. 2, p. 117-123, 2018.

PIMENTEL, $V$ et al. Biodiversidade brasileira como fonte da inovação farmacêutica: uma nova esperança? Revista do BNDES, v. 43, p. 41-89, 2015.

RODRIGUES, J. et al. A versatilidade no uso de óleos essenciais. In: VIANNA, U.R. et al. Tópicos especiais em ciência animal VI. 1. ed. Alegre/ES: Unicopy, 2017. p. 97-108.

TAVARES, M. et al. Trends in insect repelent formulations: A review. Internacional Journal of Pharmaceutical. v. 539, p. 190-209, 2018.

WHO, 2013. Factsheet on the World Malaria Report 2013. (December 2013, Geneva).

WYLIE, B. J. et al. Insect Repellants During Pregnancy in the Era of the Zika Virus. Obstet Gynecol, v. 128, n. 5, p. 1.1111.115, 2016. 